\title{
Crystal structure of (1,10-phenanthroline- $\left.\kappa^{2} N, N^{\prime}\right)$ oxo $(N$-salicylidene- isoleucinato- $\left.\kappa^{3} O, N, O^{\prime}\right)$ vanadium(IV), $\operatorname{VO}\left(\mathrm{C}_{12} \mathrm{H}_{8} \mathrm{~N}_{2}\right)\left(\mathrm{C}_{13} \mathrm{H}_{15} \mathrm{NO}_{3}\right)$
}

\author{
L.-Z. Li*, B.-Q. Jing, L.-W. Li and T. Xu \\ Liaocheng University, School of Chemistry and Chemical Engineering, Liaocheng, Shandong 252059, P. R. China
}

Received October 5, 2006, accepted and available on-line December 15, 2006; CCDC no. 1267/1907

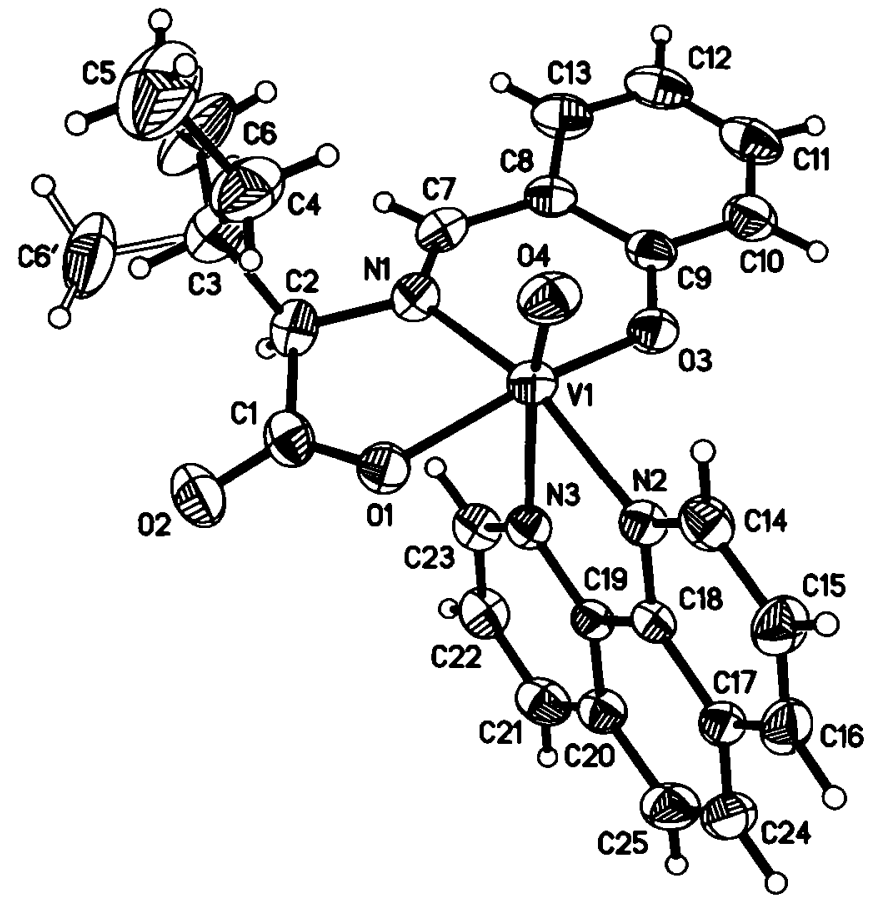

Abstract

$\mathrm{C}_{25} \mathrm{H}_{23} \mathrm{~N}_{3} \mathrm{O}_{4} \mathrm{~V}$, trigonal, $R \overline{3}$ (no. 148), $a=32.59(1) \AA$, $c=10.974(4) \AA, V=10096 \AA^{3}, Z=18, R_{\mathrm{gt}}(F)=0.053$, $w R_{\text {ref }}\left(F^{2}\right)=0.152, T=293 \mathrm{~K}$.

\section{Source of material}

Salicylaldehyde $(2 \mathrm{mmol}, 0.21 \mathrm{ml})$ was dissolved in hot ethanol $(10 \mathrm{ml})$ and was added slowly to the mixture of $\mathrm{D}, \mathrm{L}$-isoleucine ( $2 \mathrm{mmol}, 262.4 \mathrm{mg}$ ) and potassium hydroxide ( $2 \mathrm{mmol}, 112 \mathrm{mg}$ ). After stirring for $1 \mathrm{~h}$, this mixture was added successively to the solution of vanadyl sulfate hydrate $(2 \mathrm{mmol}, 451 \mathrm{mg}$ ) in $4 \mathrm{ml}$ water and further refluxed for $4 \mathrm{~h}$. Subsequently, ethanol solution of 1,10-phenanthroline monohydrate ( $2 \mathrm{mmol}, 396 \mathrm{mg}$ ) was dropped gradually into to the reaction mixture and refluxed for $3 \mathrm{~h}$, obtaining a deep yellow solution. It was filtered and held at room temperature for seven days, whereupon yellow crystals suitable for X-ray diffraction were obtained.

\footnotetext{
* Correspondence author (e-mail: lilianzhi1963@yahoo.com.cn)
}

\section{Discussion}

Vanadium is a biologically essential trace element, encountered in metalloenzymes such as haloperoxidases or nitrogenases. Since vanadyl cation has been tested as a component of oral insulin-mimetic substances in humans [1], the coordination chemistry of oxovanadium (IV) (i.e. $\mathrm{VO}^{2+}$ or vanadyl) is currently of great interest. But compared with other transition metal complexes, less vanadium complexes have been synthesized and characterized. In the course of studying oxovanadium(IV) complexes $[2,3]$, a V(IV) complex with 1,10-phenanthroline and a tridentate Schiff base ligand derived from salicylaldehyde and D,L-isoleucine was synthesized and its crystal structure determined.

In the title complex, the central vanadium(IV) atom is in a distorted octahedral environment with six ligand atoms. The terminal oxo $\mathrm{O} 4$ and the $\mathrm{N} 3$ atom from 1, 10-phenanthroline occupy the axial positions with an $04-\mathrm{V} 1-\mathrm{N} 3$ angle of $167.6(2)^{\circ}$. The $\mathrm{N} 2$ atom from the phenanthroline and the $\mathrm{N} 1, \mathrm{O} 1$ and $\mathrm{O} 3$ atoms from the tridentate Schiff base form the equatorial plane of the coordination polyhedron. The $\mathrm{V}^{\mathrm{IV}}$ atom is displaced towards the 04 by 0.291 (2) $\AA$ from the equatorial plane. The $V=0$ distance of 1.585 (3) $\AA$ is very close to that value reported for another six-coordinated Schiff base oxovanadium(IV) complex, [ $\left.\mathrm{V}\left(\mathrm{C}_{17} \mathrm{H}_{15} \mathrm{NO}_{5}\right) \mathrm{O}\left(\mathrm{C}_{10} \mathrm{H}_{8} \mathrm{~N}_{2}\right)\right] \cdot \mathrm{CH}_{3} \mathrm{OH} \cdot \mathrm{H}_{2} \mathrm{O}$ [2], which is characteristic for oxovanadium(IV) complexes $(1.588 \AA-1.601 \AA)$ [1]. The V1 -01 and the V1-O3 bond distances, $1.980(3) \AA$ and $1.921(3) \AA$, respectively, are in the range observed for many vanadium Schiff base complexes $[1,3-5]$ and due to the Jahn-Teller effect. The Schiff base forms two chelating rings to the vanadium atom, O1, C1, C2, N1, V1 and N1, C7 - C9, O3, V1, which have the dihedral angle $12.3(2)^{\circ}$ and $7.5(2)^{\circ}$ to the equatorial plane, respectively. The least-squares plane of 1,10 -phenanthroline ligand is almost perpendicular to the equatorial plane (dihedral angle $88.47(7)^{\circ}$. In the molecule, pairs of 1,10 -phenanthroline ligands related by the symmetry center have ring centroid-centroid separations of $3.42 \AA$ and $3.45 \AA$ (perpendicular distance $3.58 \AA$ ) for the N2, C14 - C18 and C17 - C20, C24, C25 rings, respectively, indicating significant $\pi-\pi$ interactions [6].

Table 1. Data collection and handling.

\section{Crystal:}

Wavelength:

$\mu$ :

Diffractometer, scan mode

$2 \theta_{\max }$ :

$N(h k l)_{\text {measured }}, N(h k l)_{\text {unique: }}$

Criterion for $I_{\mathrm{obs}}, N(h k l)_{\mathrm{gt}}$ :

$N(\text { param })_{\text {refined: }}$

Programs: yellow block, size $0.06 \times 0.28 \times 0.32 \mathrm{~mm}$

Mo $K_{\alpha}$ radiation $(0.71073 \AA)$

$4.80 \mathrm{~cm}^{-1}$

Bruker SMART CCD, $\varphi / \omega$ $50^{\circ}$

17804,3950

$I_{\mathrm{obs}}>2 \sigma\left(I_{\mathrm{obs}}\right), 1713$

309

SHELXS-97 [7], SHELXL-97 [8] 
Table 2. Atomic coordinates and displacement parameters (in $\AA^{2}$ ).

\begin{tabular}{lllllll}
\hline Atom & Site & Occ. & $\boldsymbol{x}$ & $y$ & $z$ & $U_{\text {iso }}$ \\
\hline $\mathrm{H}(2)$ & $18 f$ & & 0.0275 & 0.1307 & 0.0989 & 0.071 \\
$\mathrm{H}(3)$ & $18 f$ & 0.54 & 0.0725 & 0.0964 & 0.1505 & 0.099 \\
$\mathrm{H}\left(3^{\prime}\right)$ & $18 f$ & 0.46 & 0.0455 & 0.1052 & 0.2776 & 0.099 \\
$\mathrm{H}(4 \mathrm{~A})$ & $18 f$ & & 0.1132 & 0.1627 & 0.3337 & 0.129 \\
$\mathrm{H}(4 \mathrm{~B})$ & $18 f$ & & 0.1389 & 0.1598 & 0.2163 & 0.129 \\
$\mathrm{H}(5 \mathrm{~A})$ & $18 f$ & & 0.1057 & 0.0914 & 0.4043 & 0.250 \\
$\mathrm{H}(5 \mathrm{~B})$ & $18 f$ & & 0.1592 & 0.1278 & 0.3789 & 0.250 \\
$\mathrm{H}(5 \mathrm{C})$ & $18 f$ & & 0.1299 & 0.0871 & 0.2853 & 0.250 \\
$\mathrm{H}(6 \mathrm{~A})$ & $18 f$ & 0.54 & 0.0009 & 0.0666 & 0.2488 & 0.211 \\
$\mathrm{H}(6 \mathrm{~B})$ & $18 f$ & 0.54 & 0.0265 & 0.0998 & 0.3601 & 0.211 \\
$\mathrm{H}(6 \mathrm{C})$ & $18 f$ & 0.54 & 0.0377 & 0.0598 & 0.3252 & 0.211 \\
$\mathrm{H}\left(6 \mathrm{~A}^{\prime}\right)$ & $18 f$ & 0.46 & 0.0259 & 0.0592 & 0.1066 & 0.245 \\
$\mathrm{H}\left(6 \mathrm{~B}^{\prime}\right)$ & $18 f$ & 0.46 & 0.0573 & 0.0491 & 0.1963 & 0.245 \\
$\mathrm{H}\left(6 \mathrm{C}^{\prime}\right)$ & $18 f$ & 0.46 & 0.0798 & 0.0793 & 0.0785 & 0.245 \\
& & & & & &
\end{tabular}

Table 2. Continued.

\begin{tabular}{|c|c|c|c|c|c|c|}
\hline Atom & Site & Occ. & $x$ & $y$ & $z$ & $U_{\text {iso }}$ \\
\hline $\mathrm{H}(7)$ & $18 f$ & & -0.0030 & 0.1423 & 0.2838 & 0.064 \\
\hline$H(10)$ & $18 f$ & & 0.0366 & 0.3153 & 0.3948 & 0.083 \\
\hline H(11) & $18 f$ & & -0.0272 & 0.2806 & 0.5202 & 0.091 \\
\hline$H(12)$ & $18 f$ & & -0.0650 & 0.2002 & 0.5583 & 0.090 \\
\hline$H(13)$ & $18 f$ & & -0.0441 & 0.1542 & 0.4468 & 0.081 \\
\hline$H(14)$ & $18 f$ & & 0.2055 & 0.3464 & 0.2001 & 0.069 \\
\hline$H(15)$ & $18 f$ & & 0.2521 & 0.4159 & 0.0972 & 0.077 \\
\hline$H(16)$ & $18 f$ & & 0.2247 & 0.4333 & -0.0736 & 0.076 \\
\hline H(21) & $18 f$ & & 0.0074 & 0.2772 & -0.2531 & 0.077 \\
\hline H(22) & $18 f$ & & -0.0323 & 0.2081 & -0.1448 & 0.074 \\
\hline$H(23)$ & $18 f$ & & 0.0036 & 0.1966 & 0.0232 & 0.068 \\
\hline$H(24)$ & $18 f$ & & 0.1555 & 0.4110 & -0.2175 & 0.079 \\
\hline $\mathrm{H}(25)$ & $18 f$ & & 0.0814 & 0.3578 & -0.2794 & 0.083 \\
\hline
\end{tabular}

Table 3. Atomic coordinates and displacement parameters (in $\AA^{2}$ ).

\begin{tabular}{|c|c|c|c|c|c|c|c|c|c|c|c|}
\hline Atom & Site & Occ. & $x$ & $y$ & $z$ & $U_{11}$ & $U_{22}$ & $U_{33}$ & $U_{12}$ & $U_{13}$ & $U_{23}$ \\
\hline $\mathrm{V}(1)$ & $18 f$ & & $0.10850(3)$ & $0.25443(3)$ & $0.18071(7)$ & $0.0474(6)$ & $0.0526(6)$ & $0.0441(5)$ & $0.0236(5)$ & $0.0021(4)$ & $0.0045(4)$ \\
\hline$N(1)$ & $18 f$ & & $0.0554(1)$ & $0.1865(1)$ & $0.2112(4)$ & $0.042(3)$ & $0.051(3)$ & $0.058(3)$ & $0.023(2)$ & $0.005(2)$ & $0.004(2)$ \\
\hline $\mathbf{N}(2)$ & $18 f$ & & $0.1499(1)$ & $0.3207(1)$ & $0.0933(3)$ & $0.044(3)$ & $0.049(3)$ & $0.043(2)$ & $0.022(2)$ & $0.001(2)$ & $0.000(2)$ \\
\hline$N(3)$ & $18 f$ & & $0.0631(1)$ & $0.2567(1)$ & $0.0161(3)$ & $0.042(3)$ & $0.048(3)$ & $0.045(2)$ & $0.020(2)$ & $0.001(2)$ & $0.000(2)$ \\
\hline$O(1)$ & $18 f$ & & $0.1223(1)$ & $0.2200(1)$ & $0.0534(3)$ & $0.054(2)$ & $0.055(2)$ & $0.062(2)$ & $0.026(2)$ & $0.011(2)$ & $0.005(2)$ \\
\hline$O(2)$ & $18 f$ & & $0.0986(1)$ & $0.1528(1)$ & $-0.0450(4)$ & $0.099(3)$ & $0.089(3)$ & $0.076(3)$ & $0.056(3)$ & $0.002(2)$ & $-0.019(2)$ \\
\hline$O(4)$ & $18 f$ & & $0.1479(1)$ & $0.2630(1)$ & $0.2782(3)$ & $0.066(2)$ & $0.071(2)$ & $0.061(2)$ & $0.029(2)$ & $-0.017(2)$ & $0.007(2)$ \\
\hline$C(1)$ & $18 f$ & & $0.0950(2)$ & $0.1753(2)$ & $0.0407(5)$ & $0.056(4)$ & $0.068(4)$ & $0.062(4)$ & $0.036(3)$ & $-0.005(3)$ & $-0.010(3)$ \\
\hline $\mathrm{C}(2)$ & $18 f$ & & $0.0582(2)$ & $0.1499(2)$ & $0.1392(5)$ & $0.048(3)$ & $0.051(3)$ & $0.078(4)$ & $0.025(3)$ & $0.000(3)$ & $-0.001(3)$ \\
\hline$C(3)$ & $18 f$ & & $0.0689(2)$ & $0.1163(2)$ & $0.2116(6)$ & $0.084(5)$ & $0.073(5)$ & $0.095(5)$ & $0.042(4)$ & $0.008(4)$ & $0.017(4)$ \\
\hline$C(4)$ & $18 f$ & & $0.1144(3)$ & $0.1410(2)$ & $0.2752(6)$ & $0.121(6)$ & $0.105(6)$ & $0.105(6)$ & $0.063(5)$ & $-0.022(5)$ & $0.019(5)$ \\
\hline$c(5)$ & $18 f$ & & $0.1286(3)$ & $0.1088(3)$ & $0.3422(9)$ & $0.173(9)$ & $0.148(8)$ & $0.21(1)$ & $0.104(7)$ & $-0.064(8)$ & $-0.005(7)$ \\
\hline$C(6)$ & $18 f$ & $0.54(1)$ & $0.0300(5)$ & $0.0826(4)$ & $0.294(2)$ & $0.11(1)$ & $0.10(1)$ & $0.23(2)$ & $0.062(9)$ & $0.08(1)$ & $0.10(1)$ \\
\hline$C\left(6^{\prime}\right)$ & $18 f$ & 0.46 & $0.0569(7)$ & $0.0719(6)$ & $0.142(2)$ & $0.25(3)$ & $0.11(2)$ & $0.19(2)$ & $0.13(2)$ & $0.06(2)$ & $0.02(1)$ \\
\hline$C(8)$ & $18 f$ & & $0.0097(2)$ & $0.2055(2)$ & $0.3523(4)$ & $0.040(3)$ & $0.062(4)$ & $0.043(3)$ & $0.024(3)$ & $0.000(3)$ & $0.007(3)$ \\
\hline $\mathrm{C}(9)$ & $18 f$ & & $0.0359(2)$ & $0.2562(2)$ & $0.3367(4)$ & $0.062(4)$ & $0.070(4)$ & $0.038(3)$ & $0.042(3)$ & $-0.002(3)$ & $0.002(3)$ \\
\hline$C(10)$ & $18 f$ & & $0.0205(2)$ & $0.2825(2)$ & $0.4034(5)$ & $0.089(5)$ & $0.083(4)$ & $0.051(3)$ & $0.054(4)$ & $0.007(3)$ & $0.001(3)$ \\
\hline$C(11)$ & $18 f$ & & $-0.0172(2)$ & $0.2619(3)$ & $0.4807(5)$ & $0.080(5)$ & $0.121(6)$ & $0.047(4)$ & $0.067(5)$ & $0.010(3)$ & $-0.003(4)$ \\
\hline$C(12)$ & $18 f$ & & $-0.0407(2)$ & $0.2139(3)$ & $0.5012(5)$ & $0.059(4)$ & $0.119(6)$ & $0.045(4)$ & $0.042(4)$ & $0.013(3)$ & $0.011(4)$ \\
\hline$C(13)$ & $18 f$ & & $-0.0274(2)$ & $0.1868(2)$ & $0.4353(5)$ & $0.052(4)$ & $0.090(4)$ & $0.056(4)$ & $0.033(3)$ & $0.012(3)$ & $0.013(3)$ \\
\hline$C(14)$ & $18 f$ & & $0.1936(2)$ & $0.3527(2)$ & $0.1301(5)$ & $0.053(4)$ & $0.061(4)$ & $0.053(3)$ & $0.025(3)$ & $-0.004(3)$ & $-0.008(3)$ \\
\hline$C(15)$ & $18 f$ & & $0.2219(2)$ & $0.3949(2)$ & $0.0686(5)$ & $0.049(3)$ & $0.048(4)$ & $0.075(4)$ & $0.009(3)$ & $0.001(3)$ & $0.004(3)$ \\
\hline$C(16)$ & $18 f$ & & $0.2056(2)$ & $0.4053(2)$ & $-0.0315(5)$ & $0.057(4)$ & $0.046(3)$ & $0.076(4)$ & $0.018(3)$ & $0.023(3)$ & $0.006(3)$ \\
\hline $\mathrm{C}(17)$ & $18 f$ & & $0.1595(2)$ & $0.3736(2)$ & $-0.0729(5)$ & $0.059(4)$ & $0.048(3)$ & $0.049(3)$ & $0.028(3)$ & $0.017(3)$ & $0.007(3)$ \\
\hline $\mathrm{C}(18)$ & $18 f$ & & $0.1332(2)$ & $0.3314(2)$ & $-0.0073(4)$ & $0.053(3)$ & $0.047(3)$ & $0.038(3)$ & $0.026(3)$ & $0.008(3)$ & $-0.001(3)$ \\
\hline$C(19)$ & $18 f$ & & $0.0864(2)$ & $0.2976(2)$ & $-0.0480(4)$ & $0.049(3)$ & $0.047(3)$ & $0.037(3)$ & $0.027(3)$ & $0.007(2)$ & $0.001(2)$ \\
\hline$\alpha(20)$ & $18 f$ & & $0.0670(2)$ & $0.3073(2)$ & $-0.1493(4)$ & $0.053(3)$ & $0.061(4)$ & $0.046(3)$ & $0.034(3)$ & $-0.002(3)$ & $0.003(3)$ \\
\hline$C(21)$ & $18 f$ & & $0.0214(2)$ & $0.2723(2)$ & $-0.1853(5)$ & $0.068(4)$ & $0.091(5)$ & $0.048(3)$ & $0.051(4)$ & $-0.010(3)$ & $-0.002(3)$ \\
\hline $\mathrm{C}(22)$ & $18 f$ & & $-0.0021(2)$ & $0.2314(2)$ & $-0.1216(5)$ & $0.054(4)$ & $0.068(4)$ & $0.063(4)$ & $0.029(3)$ & $-0.008(3)$ & $-0.008(3)$ \\
\hline$C(23)$ & $18 f$ & & $0.0200(2)$ & $0.2247(2)$ & $-0.0205(5)$ & $0.052(4)$ & $0.062(4)$ & $0.054(3)$ & $0.028(3)$ & $0.000(3)$ & $-0.001(3)$ \\
\hline
\end{tabular}

Acknowledgments. The authors thank the Natural Science Foundation of Shandong Province (grant no. Y2004B02) and the National Natural Science Foundation of China (grant no. 20543004) for a research grant.

\section{References}

1. Kenji, K.; Makoto, T.; Ken, H.; Naohisa, Y.; Yoshitane, K.: Syntheses and crystal structures of oxovanadium(IV) complexes with $N, N^{\prime}$-ethylenebis(amino acids). Inorg. Chim. Acta 305 (2000) 172-183.

2 Jing, B.-Q.; Li, L.-Z.; Wang, D.-Q.; Xu, T.: $\left(2,2^{\prime}\right.$-Bipyridine- $\left.\kappa^{2} N, N^{\prime}\right)$ oxo$\left(N\right.$-vanillylidenetyrosinato- $\left.\kappa^{3} O, N, O^{\prime}\right)$ vanadium(IV) methanol solvate monohydrate. Acta Crystallogr. E61 (2005) m2106-m2108.
3. Xu, T.; Li, L.-Z.; Zhou, S.-F.; Guo, G.-Q.; Niu, M.-J.: Synthesis, characterization and crystal structure of oxovanidium(IV) complex with tridentate a-van-gly and bidentate phenanthroline. J. Chem. Crystallogr. 35 (2005) 263-267.

4. Gebray, A.; Alavattam, S.; Jussi, K.; Rao, C. P.: Synthesis and characterization of vanadyl(IV) complexes of Schiff bases derived from anthranilic 
acid and salicylaldehyde (or its derivatives) or acetyl acetone. Singlecrystal X-ray structures of the oxidized products. Polyhedron 16 (1997) 643-651.

5. Root, C. A.; Hoeschele, J. D.; Comman, C. R.; Kampf, J. W.; Pecoraro, V. L.: Structural and spectroscopic characterization of dioxovanadium(V) complexes with asymmetric Schiff base ligands. Inorg. Chem. 32 (1993) 3855-3861.

6. Tong, M. L.; Lee, H. K; Chen, X. M.; Huang, R. B.; Mak, T. M. C.: A novel three-dimensional triangular organic-inorganic hybrid network selfassembled by mononuclear $\left[\mathrm{Mn}\left(4,4^{\prime} \text {-bipyridine }\right)_{2}\left(\mathrm{H}_{2} \mathrm{O}\right)_{4}\right]^{2+}$ cations and rich solvate 4,4' -bipyridine molecules through bydrogen-bonding and interactions. J. Chem. Soc., Dalton. Trans. 39 (1999) 3657-3659.

7. Sheldrick, G. M.: SHELXS-97. Program for the Solution of Crystal Structures. University of Göttingen, Germany 1997.

8. Sheldrick, G. M.: SHELXL-97. Program for the Refinement of Crystal Structures. University of Göttingen, Germany 1997. 